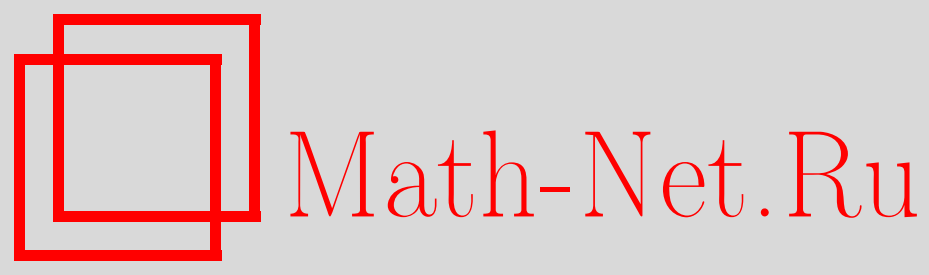

М. А. Чахкиев, Вопрос о точном значении одностороннего поперечника остается открытым, Матем. заметки, 2016, том 100, выпуск 2, 318-320

DOI: https://doi.org/10.4213/mzm11223

Использование Общероссийского математического портала Math-Net.Ru подразумевает, что вы прочитали и согласны с пользовательским соглашением http://www . mathnet.ru/rus/agreement

Параметры загрузки:

IP : 54.157 .27 .8

26 апреля 2023 г., 14:41:11

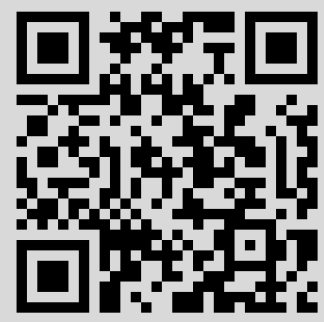




\section{Вопрос о точном значении одностороннего поперечника остается открытым}

\section{М. А. Чахкиев}

Ключевые слова: поперечники периодических классов, котрпример.

DOI: $10.4213 / \mathrm{mzm} 11223$

В книге [1; гл. 10, § 10.4] рассматриваются односторонние поперечники периодических классов $W_{\infty}^{r}$. Однако основное, как сказано в книге, утверждение этого параграфа - лемма 10.4.1 на с. 230 - неверно. В самой книге лемма приведена без доказательства. Лемма принадлежит Рубану [2], однако доказательство, где строится некоторое специальное отображение и подсчитывается его степень, приведено лишь схематично.

У автора были сомнения по поводу целесообразности публикования котрпримера к лемме, однако выяснилось, что специалистам в теории приближений неизвестно о неверности леммы. Более того, предпринимаются попытки к ее обобщению. Прежде чем рассмотреть формулировку леммы и контрпример к ней, приведем некоторые необходимые определения и обозначения из [1].

Пусть $X$ - некоторое линейное нормированное пространство вещественнозначных функций $x(t)$, заданных на отрезке $[0,2 \pi]$. Величину

$$
E^{+}(x, K)=\inf \{\|x-u\|: u(t) \geqslant x(t), u \in K\}
$$

называют наилучшим приближением сверху функции $x(t)$ множеством $K$ в метрике пространства $X$. Наилучшим приближением сверху множества $L$ множеством $K$ в метрике пространства $X$ называют величину

$$
E^{+}(L, K)=\sup _{x \in L} E^{+}(x, K) .
$$

Величину

$$
d_{n}^{+}(L)=\inf _{\substack{K C X \\ \operatorname{dim} K=n}} E^{+}(L, K),
$$

где нижняя грань берется по всем $n$-мерным подпространствам пространства $X$, называют односторонним (сверху) поперечником множества $L$.

Лемма (Рубан [2]). Пусть $M_{2 n}=\operatorname{span}\left\{1, e_{2}, e_{3}, \ldots, e_{2 n}\right\}$,

$$
u^{+}(x)=\lambda_{1}(x)+\lambda_{2}(x) e_{2}+\cdots+\lambda_{2 n}(x) e_{2 n}
$$

- функиия наилучшего приближения сверху для $x$ в пространстве $M_{2 n}$ в метрике $L_{1}$. Тогда найдется функиия $x^{+} \in q_{n, 1}$ такая, что

$$
\lambda_{1}\left(x^{+}\right) \geqslant \max _{t} x^{+}(t) .
$$

Здесь $e_{2}, \ldots, e_{2 n}$ - периодические с периодом $2 \pi$ функции со средним значением на периоде равным нулю, $q_{n, 1}-$ ломаные с не более чем $2 n$ звеньями, каждое из которых наклонено к оси под углом $\pi / 4$ или $3 \pi / 4$.

Перейдем к построению контрпримера к лемме. Разобьем отрезок $[0,2 \pi]$ на $2 n-1$ равных интервала

$$
\left(\frac{2 \pi(k-2)}{2 n-1}, \frac{2 \pi(k-1)}{2 n-1}\right), \quad k=2,3, \ldots, 2 n,
$$

DOI: $10.4213 / \mathrm{mzm} 11223$

(C) М. А. ЧАхкиев, 2016 
и пусть $v_{k}=v_{k}(t)$ функция, равная нулю на периоде вне интервала

$$
\left(\frac{2 \pi(k-2)}{2 n-1}, \frac{2 \pi(k-1)}{2 n-1}\right)
$$

равная единице в середине интервала и линейная на двух оставшихся участках.

Пусть $M_{2 n}=\operatorname{span}\left\{1, e_{2}, e_{3}, \ldots, e_{2 n}\right\}$, где $e_{k}=v_{k}-\pi /(2 n-1), k=2, \ldots, 2 n$, так что среднее значение на периоде функций $e_{2}, \ldots, e_{2 n}$ равно нулю. Если

$$
u^{+}(x)=\lambda_{1}(x)+\lambda_{2}(x) e_{2}+\cdots+\lambda_{2 n}(x) e_{2 n}
$$

- функция наилучшего приближения сверху для $x$ в пространстве $M_{2 n}$ в метрике $L_{1}$, то по лемме найдется функция $x^{+} \in q_{n, 1}$ такая, что $\lambda_{1}\left(x^{+}\right) \geqslant \max _{t} x^{+}(t)$.

Без ограничения общности будем считать, что $\max _{t} x^{+}(t)=0$. Пусть $t_{1}, t_{2}, \ldots, t_{m}$ точки локального максимума функции $x^{+}(t)$. Так как $x^{+}(t)$ ломаная с не более чем $2 n$ звеньями, то $m \leqslant n$. Если $2 n-1>n$, т.е. $n \geqslant 2$, то найдется интервал с номером $j$,

$$
\left(\frac{2 \pi(j-2)}{2 n-1}, \frac{2 \pi(j-1)}{2 n-1}\right)
$$

такой, что ни одна из точек $t_{1}, t_{2}, \ldots, t_{m}$ не принадлежит этому интервалу. Тогда нетрудно видеть, что в силу определения функции $v_{j}$ и вида функции $x^{+}(t)$ выполнена оценка $-\alpha v_{j}(t)-x^{+}(t) \geqslant 0$, где положительное число $\alpha$ выбрано так, что производная функции $\alpha v_{j}(t)$ по модулю меньше единицы. Отсюда

$$
\begin{aligned}
& \int_{0}^{2 \pi}\left(-\alpha v_{j}(t)-x^{+}(t)\right) d t \\
& \quad=\int_{0}^{2 \pi}\left(\alpha\left(-e_{j}-\frac{\pi}{2 n-1}\right)-x^{+}(t)\right) d t=-\int_{0}^{2 \pi} x^{+}(t) d t-\frac{2 \alpha \pi^{2}}{2 n-1} .
\end{aligned}
$$

Далее,

$$
\int_{0}^{2 \pi}\left(u^{+}(x)-x^{+}(t)\right) d t=2 \pi \lambda_{1}\left(x^{+}\right)-\int_{0}^{2 \pi} x^{+}(t) d t .
$$

Так как $\lambda_{1}\left(x^{+}\right) \geqslant \max _{t} x^{+}(t)=0$, то из (1) и (2) следует

$$
\int_{0}^{2 \pi}\left(-\alpha v_{j}(t)-x^{+}(t)\right) d t<\int_{0}^{2 \pi}\left(u^{+}(x)-x^{+}(t)\right) d t,
$$

что противоречит тому, что функция $u^{+}(x)=\lambda_{1}(x)+\lambda_{2}(x) e_{2}+\cdots+\lambda_{2 n}(x) e_{2 n}$ - наилучшее приближение сверху для $x^{+}(t)$ в пространстве $M_{2 n}$ в метрике $L_{1}$.

Полученное противоречие показывает, что лемма не верна уже при $n \geqslant 2$.

На основе леммы в [2] устанавливаются точные оценки односторонних поперечников классов периодических функций $W_{\infty}^{r}, W^{r} C$ :

$$
d_{2 n}^{+}\left(W_{\infty}^{r}\right)_{1}=d_{2 n-1}^{+}\left(W_{\infty}^{r}\right)_{1}=d_{2 n}^{+}\left(W^{r} C\right)_{1}=d_{2 n-1}^{+}\left(W^{r} C\right)_{1}=2 \pi \frac{K_{r}}{n^{r}}, \quad n, r=1,2, \ldots,
$$

которые, таким образом, нельзя считать доказанными.

Отметим еще, что лемма была бы верна, если функции $1, e_{2}, e_{3}, \ldots, e_{2 n}$ образовывали систему функций Чебышева на периоде. Для всех известных случаев экстремальные подпространства (многочлены, тригонометрические многочлены, сплайны) оказывались чебышевскими системами. В связи с этим было бы интересно выяснить, для каких выпуклых, центрально-симметричных, компактных классов гладких функций среди экстремальных подпространств есть чебышевские системы. 


\section{СПИСОК ЦИТИРОВАННОЙ ЛИТЕРАТУРЫ}

[1] Н. П. Корнейчук, А. А. Лигун, В. Г. Доронин, Аппроксимачия с ограничениями, Наукова думка, Киев, 1982. [2] В. И. Рубан, ДАН СССР, 225:1 (1980), 34-35.

М. А. Чахкиев

Российский государственный

социальный университет, г. Москва

E-mail: chahkiev_magomed@mail.ru
Поступило

21.12 .2015 